\title{
Mediation as a strategy for constructing meaning in the teaching of a subject by integrating a foreign language
}

\section{[La médiation au service de la construction du sens dans l'enseignement d'une matière par l'intégration d'une langue étrangère]}

\author{
Magdalena Sowa - Joanna Kic-Drgas
}

DOI: 10.18355/XL.2021.14.04.18

\begin{abstract}
The increasing globalization as well as internationalization have influenced educational systems all over the world, contributing to widespread of the idea of teaching in a second or foreign language so as to allow students, ranging from primary to tertiary levels, to compete in an international context. The aim of this paper is to analyse and categorise the strategies of mediation used for presentation of subject content knowledge while teaching in bilingual French-Polish classes at secondary school level. In order to take the complex nature of the issue into account, variety of used strategies will be examined by means of corpus analysis. The material used for analysis comes from the bilingual lessons of chemistry and biology recorded in secondary school in Warsaw in the period between November and December 2019. The studies presented will allow conclusions to be drawn regarding the potential possibilities and problems of content-knowledge presentation in foreign langue.
\end{abstract}

Key words: CLIL, bilingual classes, content-subject knowledge, teacher, mediation

\section{Résumé}

La mondialisation croissante a influencé les systèmes éducatifs du monde entier. Des processus globaux ont contribué à la propagation de l'enseignement assuré dans une langue seconde ou étrangère pour permettre aux étudiants, du primaire au supérieur, de rivaliser dans un contexte international. L'objectif de cet article est d'analyser et de catégoriser les stratégies de médiation utilisées pour la présentation et l'explicitation des contenus disciplinaires dans des classes bilingues franco-polonaises au niveau secondaire. Afin de prendre en compte la nature complexe de la problématique, la variété des stratégies utilisées sera examinée à partir d'une analyse de corpus. Les données empiriques sont issues des cours bilingues de chimie et de biologie enregistrés au lycée de Varsovie entre novembre et décembre 2019. L'interprétation des données et la discussion des résultats permettront de tirer des conclusions sur les possibilités/modalités de la construction du sens dans l'enseignement d'une matière par l'intégration d'une langue étrangère et de problèmes potentiels qui en résultent.

Mots clés : EMILE, classes bilingues, savoir disciplinaire, enseignant, médiation

\section{Introduction}

Le plurilinguisme est l'un des phénomènes majeurs qui influencent le système d'éducation européen. Son impact est particulièrement perceptible dans la politique linguistique de l'Union Européenne, qui vise à promouvoir toute initiative relative au développement de la compétence de communication en plusieurs langues, mais aussi dans les actions des États-membres qui cherchent à renforcer la présence des langues étrangères (LE) dans les programmes d'enseignement nationaux. Ces initiatives sont sollicitées par les défis de la société globale où la connaissance des LE permet aussi aux apprenants de développer leurs savoir-faire en langue maternelle et d'accéder aux échanges scientifiques, artistiques et/ou professionnels avec les interlocuteurs du monde entier. L'enseignement d'une matière scolaire en langue étrangère constitue l'une des options censées préparer les utilisateurs de la langue à faire face aux enjeux 
sociaux et professionnels de la société moderne. Connu entre autres sous le nom d'EMILE (Enseignement d'une matière par l'intégration d'une langue étrangère), de CLIL (Content and Language Integrated Learning) ou tout simplement comme enseignement bilingue ou disciplinaire ${ }^{1}$, cet enseignement vise l'appropriation de deux objets de connaissance : le savoir disciplinaire et la langue étrangère. Construit sur une forte idéologie européenne et adopté dans de nombreux pays, il met en exergue le rôle de la langue étrangère (LE) ou seconde (L2) en tant qu'outil pédagogique.

Bien que l'EMILE soit pratiqué depuis plusieurs années dans les écoles européennes, il ne dispose pas pour autant d'une véritable théorie (Carol, 2015). Afin de rendre compte de sa spécificité, il importe de capter les pratiques de classe et de les expliquer $\mathrm{du}$ point de vue des actions accomplies par l'enseignant et les apprenants. L'observation directe des activités visant l'appropriation de deux objets de connaissance s'avère donc cruciale pour l'analyse de divers paramètres de la situation pédagogique propre à ce type d'enseignement et pour sa théorisation. Étant donnée la complexité du problème, qui est par essence transdisciplinaire, le présent texte entreprend une réflexion plus modeste et centrée sur l'enseignant d'une discipline non linguistique. Puisque l'enseignement bilingue repose sur la communication entre « un expert» et «un novice » et comme les savoirs s'acquièrent, entre autres, par l'écoute et le discours, il importe de s'interroger sur les moyens mobilisés par l'enseignant non seulement pour un transfert d'informations, mais surtout pour la construction des savoirs par et chez l'apprenant. Notre objectif vise donc à décrire les gestes professionnels (ou les stratégies de médiation) ${ }^{2}$ que l'enseignant utilise pour transmettre les savoirs et expliciter les contenus liés à la discipline et la langue.

La réflexion, que nous proposons dans le présent texte, prend appui sur l'observation directe des gestes professionnels de médiation adoptés par des enseignants lors des cours de chimie et de biologie assurés en français langue étrangère (FLE). Afin d'en rendre compte, nous avons enregistré une trentaine de cours de trois enseignants polonais chargés de l'EMILE en français à des adolescents polonais fréquentant un lycée bilingue de Varsovie. Les transcriptions des leçons enregistrées constituent un corpus considérable des données empiriques recueillies en 2019 dont nous nous servirons en vue d'expliciter les stratégies de médiation utilisées par les enseignants en question au service de la transmission des savoirs, et plus concrètement de l'explication des nouveaux concepts.

\section{EMILE/CLIL - une approche difficile à circonscrire ?}

Content and Language Integrated Learning consiste à enseigner une matière (soit des contenus disciplinaires) en langue étrangère ou seconde et, le plus souvent, intervient dans les programmes d'enseignement des sciences exactes (comme les mathématiques, la chimie, la biologie, la géographie) ou sciences humaines (p.ex. l'histoire). Orientée sur le contenu, l'approche EMILE/CLIL se situe à la fois comme

\footnotetext{
${ }^{1}$ Nous considérons tous ces termes comme synonymiques et nous les utiliserons au cours de notre réflexion en y attribuant le sens égal.

${ }^{2}$ Selon M. Pégourié-Khellef (2019), le terme de «geste professionnel » convient mieux pour décrire les activités d'enseignement du point langagier, méta-langagier et métalinguistique, les stratégies n'analysant ces activités que d'un point de vue cognitif et métacognitif. Les gestes professionnels de médiation relèvent forcément de stratégies de médiation (Conseil de l'Europe, 2001, 2018). Dans notre réflexion, nous utiliserons les deux termes (geste professionnel de médiation et stratégie de médiation) pour décrire le mode d'action adopté par l'enseignant au profit de l'approche EMILE/CLIL.
}

XLinguae, Volume 14 Issue 4, October 2021, ISSN 1337-8384, eISSN 2453-711X 
expérience d'un apprentissage de langue tout en se distinguant des approches d'enseignement de la langue existantes (Coyle, Hood, Marsh, 2010). Cependant, il importe de souligner qu'elle ne prétend pas à une nouvelle forme d'enseignement de langue ni de contenu, mais elle constitue une innovante fusion des deux.

Comme l'EMILE/CLIL combine les aspects linguistiques et disciplinaires, il poursuit naturellement un double objectif : celui de permettre aux élèves «d'acquérir des connaissances dans des contenus spécifiques du programme d'études » et celui de les amener à « développer des compétences linguistiques dans une autre langue que celle utilisée habituellement comme une langue d'instruction » (Eurydice, $2006: 22$ ).

S'il est possible de considérer l'EMILE/CLIL comme un modèle où le contenu et la langue ont une importance égale, les chercheurs ne sont pas unanimes dans la façon de définir cette approche, ce dont témoignent les définitions ci-dessous :

"[CLIL] is a dual-focused educational approach in which an additional language is used for learning and teaching of both content and language. There is a focus not only on content and not only on language. Each is interwoven ${ }^{3}-$ even if the emphasis is greater on one than the other at a given time" (Coyle, Hood, Marsh, 2010: $1)$.

"CLIL can be described as an educational approach where curricular content is taught through the medium of a foreign language, typically to students participating in some form of mainstream ${ }^{4}$ education at the primary, secondary, or tertiary level" (Dalton-Puffer, 2011: 183).

"CLIL embraces those educational practices in which content subjects - excluding those labelled as 'language subjects' - are taught and learned through a language of instruction, second or foreign, in which a learner has a basic or advanced developing communicative competence, and which explicitly:

- Promote the preservation and development of the learner's first language(s) and the consideration of and mise en valeur of cultural forms attached to that (those) language(s);

- Promote a truly integrated approach, with a dual focus of pedagogical attention, i.e., language and content; and

- Provide learners with all the assistance needed to comprehend, produce and negotiate academic messages in the target language adopted as the medium of instruction" (Escobar Urmeneta, 2011: 203-204).

La définition de D. Cole et al. (2010) fait ressortir au premier plan la co-présence des contenus linguistiques et disciplinaires. Celle de Ch. Dalton-Puffer (2011) insiste davantage sur l'environnement dans lequel l'enseignement de la discipline non linguistique en LE a lieu. C. Escobar Urmeneta (2011) ponctue en revanche les bénéfices de la mise en pratique du CLIL tout en soulignant leur dimension pratique (educational practices).

En outre de la diversité d'éléments pris en compte dans les définitions disponibles de l'approche en question, il faut également noter la prolifération ${ }^{5}$ des termes utilisés pour dénommer ce type d'enseignement. Seule la littérature en français et anglais

\footnotetext{
${ }^{3}$ Souligné par les auteures.

${ }^{4}$ Souligné par les auteures.

${ }^{5}$ Selon O. García et H. Baetens Beardsmore (2009 : 208), plus de 30 dénominations différentes sont en vigueur pour désigner cette méthodologie éducative où la langue étrangère constitue un outil pour apprendre/enseigner une discipline.
} 
parle de l'enseignement d'une matière par l'intégration d'une LE/L2, l'enseignement d'une discipline non linguistique (DNL), l'enseignement bilingue, l'enseignement disciplinaire, l'approche immersive, la méthode intégrée, Content-Based Instruction (CBI), Content Based Language Learning and Teaching, Language-medium instruction. Même si toutes ces conceptions sont subordonnées à un objectif commun, à savoir le bi- ou plurilinguisme résultant de l'appropriation simultanée de la langue et la discipline, la multitude de dénominations contribue souvent à la confusion terminologique et aux mésententes entre les didacticiens. Dans le contexte anglosaxon par exemple, J. Cenoz et al. (2010) considèrent le CLIL comme un hyperonyme englobant différentes autres approches et notions, alors que Ch. Dalton-Puffer et al. (2014) opposent nettement le CLIL au CBI, les deux visant respectivement l'enseignement du contenu disciplinaire (CLIL) et l'enseignement de la langue $(\mathrm{CBI})^{6}$.

\section{EMILE/CLIL et ses principes}

Le CLIL repose sur quatre principes identifiés par D. Coyle (2006) qui constituent un cadre 4C, à savoir le contenu (content), la communication (communication), la cognition (cognition) et la culture (culture).

Le premier principe de ce cadre renvoie au contenu qui demeure le point central de l'apprentissage/enseignement. Il est notamment question de la compréhension et de l'acquisition des savoirs et savoir-faire relatifs à la discipline en question. La relation entre la langue et l'appréhension de la discipline requiert de s'interroger sur la façon d'enseigner les contenus disciplinaires tout en travaillant avec et à travers la langue étrangère plutôt que dans la langue étrangère.

La communication, étant le deuxième principe, consiste non seulement à apprendre à utiliser une langue étrangère, mais aussi à utiliser celle-ci pour apprendre. Dans cette perspective, la langue constitue à la fois un outil de communication et d'apprentissage, ce qui contraint à l'utiliser/apprendre dans des situations authentiques et nouvelles. L'idée développée par l'approche EMILE/CLIL est d'insister sur le statut de la langue en tant qu'outil qui, pour avoir du sens, doit être activée dans des situations motivantes et ayant du sens pour les apprenants.

Le troisième principe, la cognition, met en avant les processus d'apprentissage et de réflexion. L'acquisition des connaissances et compétences est sans doute importante, mais il importe aussi de mettre l'accent sur la méthode d'apprentissage (la résolution des problèmes, la réflexion etc.), celle-ci ne se limitant pas à un simple transfert de savoirs et/ou savoir-faire. L'idée centrale de l'approche EMILE/CLIL est de donner lieu à un apprentissage personnalisé et une compréhension individuelle.

Enfin, le quatrième principe - la culture - «permet de promouvoir une compréhension interculturelle en reliant l'apprentissage d'une matière et celui d'une langue. À travers cette vision d'un environnement plurilingue et pluriculturel, l'apprenant peut accéder à une compréhension du monde, et à une interprétation des événements suivant des perspectives variées, ce qui serait impossible dans une approche monolingue » (Rodrigues, Wigham, 2013 : non paginé).

Pour R. Gozdawa-Golebiowski et al. (2019), les principes fondamentaux de l'EMILE/CLIL résident dans la progression communicative et conceptuelle,

\footnotetext{
${ }^{6}$ Il en va de même pour le contexte polonais où les chercheurs-didacticiens sont loin de se mettre d'accord sur les questions terminologiques (Olpinska-Szkielko, 2008).
}

XLinguae, Volume 14 Issue 4, October 2021, ISSN 1337-8384, eISSN 2453-711X 
l'activation du savoir, le feed-back et l'incitation à la réflexion, la coopération, la résolution des problèmes et un apprentissage actionnel, l'autoévaluation soutenue par l'évaluation formative. L'appropriation du savoir disciplinaire permet aux apprenants de développer leurs capacités de restructuration cognitive (telles que l'observation, la prise de conscience, la remise en question des schémas cognitifs etc.) et de compréhension. La progression conceptuelle requiert que l'enseignant mobilise des outils linguistiques adéquats afin d'assurer la synergie entre l'apprentissage des contenus relatifs à la discipline et à la langue. De plus, de nouvelles informations disciplinaires devraient prendre appui sur les éléments du savoir déjà intégrés et bien consolidés dans le répertoire des connaissances des apprenants. L'activation du savoir nécessaire pour l'appropriation de nouveaux contenus se réalise à travers des représentations et grâce au recours à des images et objets. Cependant, il importe également d'insister sur les activités et exercices que l'enseignant crée et/ou utilise pour amener ses apprenants à faire usage du savoir acquis et pour les inciter à la réflexion sur ce qu'ils sont en train d'apprendre. C'est pourquoi il est recommandé que les actions de l'enseignant ainsi que les activités pédagogiques proposées dans le cadre de l'approche EMILE/CLIL visent un apprentissage actionnel et coopératif, tout en privilégiant l'autonomie, l'éveil de la curiosité et la motivation pour l'apprentissage. Enfin, l'approche EMILE/CLIL postule à maintenir un engagement personnalisé de l'apprenant et sa disponibilité à construire le savoir jusqu'à la fin de l'apprentissage, ce qui s'avère possible grâce aux modalités d'évaluation où il y a de la place pour la réflexion, l'analyse et la prise de conscience individuelle sur les résultats du processus pédagogique.

Il s'ensuit de ce qui précède que la démarche adoptée dans le cadre de l'EMILE/CLIL doit s'orienter à la fois sur la langue et la discipline, tout en incitant les apprenants à un apprentissage actif et les engageant à la construction des savoirs disciplinaires et savoir-faire langagiers. Afin de rendre compte de la présence et du rôle de la langue dans un cours de discipline ainsi que de l'implication de l'apprenant dans un processus cognitif avancé, il est nécessaire de réfléchir plus profondément sur les compétences de l'enseignant et sa façon d'agir en classe.

\section{Le profil de l'enseignant EMILE/CLIL}

Il est évident que le rôle de l'enseignant est très important dans l'approche EMILE/CLIL. Cependant, de nombreuses interrogations se posent quant au profil de l'enseignant qui prend en charge ce type d'enseignement et quant au rapport que l'enseignement bilingue a avec le vécu professionnel des enseignants de discipline. L'EMILE/CLIL relève-il en premier lieu d'un domaine disciplinaire ou d'un domaine linguistique ? L'EMILE/CLIL devrait-il être assuré par un enseignant de langue ou un enseignant de discipline qui enseigne en LE ? Nous sommes d'accord avec M. Pégourié-Khellef (2019: 147) que «la légitimité disciplinaire joue en faveur des enseignants de discipline ; (..) il nous semble difficile, voire peu recommandé, (...) qu'un enseignant de langue se lance dans l'enseignement d'un contenu disciplinaire sans avoir été formé d'un point de vue académique à cette discipline. L'enseignement de contenu disciplinaire ne peut se réduire à une approche uniquement portée par la dimension linguistique ». D. Marsh et B. Marsland (1999: 38) sont d'avis que, en fonction des compétences des enseignants, l'accent peut être davantage mis soit sur la compétence linguistique soit sur la compétence disciplinaire ; néanmoins, le double intérêt et la double capacité, sinon la double qualification, semblent être au plus haut point souhaitables.

Il reste cependant notoire que la gamme de compétences dont l'enseignant devrait faire preuve dépasse largement celles qui se réfèrent à la discipline et la langue. Parmi les caractéristiques associées à l'enseignant chargé de l'enseignement bilingue, G. 
Whitty (1996 : 89-90) énumère la volonté de perfectionner ses savoirs et savoir-faire professionnels, les compétences de communication, le savoir disciplinaire, la compréhension pour les élèves. D. Marsh et al. (2001) développent davantage ce répertoire de compétences idéales et y inscrivent les compétences de communication aussi bien en LE/L2 qu'en L1, le savoir en didactique des langues, le savoir-faire pédagogique, la connaissance de son milieu éducatif, la capacité à concevoir et exploiter les supports pédagogiques et à évaluer le progrès des apprenants.

Un point de vue intéressant sur les compétences des enseignants EMILE/CLIL, qui détaille les idées susmentionnées, est présenté par D. Wolff (2002). Ce dernier constate que le profil professionnel de l'enseignant assurant ses cours dans l'approche EMILE/CLIL ne doit pas être envisagé comme une simple somme de compétences propres à un enseignant de langue et à celui d'une matière non-linguistique. Selon cette perspective, la didactique des DNL en LE/L2 constitue un domaine à part qui diffère de la didactique des autres matières singulières. Ceci étant, en identifiant les savoirs et savoir-faire requis chez l'enseignant chargé de l'enseignement bilingue, Wolff (2002 : 145-146) recommande que ce dernier soit, entre autres, capable de/d' :

- communiquer en LE au niveau proche de celui des locuteurs natifs ;

- $\quad$ varier les styles linguistiques, en particulier en ce qui concerne la langue de spécialité/discipline en question ;

- comprendre les opérations propres au traitement des informations lors des activités de réception et de production en LE ;

- faire usage des théories relatives à l'acquisition des langues et au bilinguisme ;

- recourir aux principes méthodologiques de la didactique des langues de spécialité ;

- reconnaître les aspects de la didactique des LE dans la didactique d'une discipline non-linguistique ;

- $\quad$ exploiter les techniques pédagogiques utilisées dans l'enseignement des LE et celui des DNL;

- utiliser des méthodes censées développer et soutenir l'autonomie de l'élève dans son apprentissage ;

- mettre en place des techniques visant le développement des activités de réception et de production en LE ;

décrire avec exactitude des phénomènes culturels liés à la discipline ;

- $\quad$ se servir des textes authentiques et ressources documentaires relatifs à la discipline.

Il convient d'en conclure que l'enseignant d'une discipline non-linguistique en LE/L2 doit connaître la langue de la discipline/spécialité qu'il enseigne, mais aussi les principes théoriques de la description de celle-ci. Ces derniers s'avèrent indispensables pour qu'il soit à même d'identifier les traits particuliers des notions et textes de spécialité, d'évaluer leur niveau de difficulté et, par conséquent, d'adopter des modes d'actions appropriés à la transmission optimale des savoirs. Ce dernier élément demeure crucial pour l'analyse que nous aborderons dans la suite de notre texte.

\section{Le rôle de la médiation dans l'approche EMILE/CLIL}

L'efficacité de l'approche EMILE/CLIL résulte en grande mesure d'une mise en place des stratégies adéquates pour la construction du sens et la transmission du savoir par l'intermédiaire de la langue étrangère. J.-L. Chiss (2012: 317) affirme qu' " [a]pprendre des disciplines, c'est donc, au moins en partie, acquérir d'autres langues, d'autres langages, d'autres moyens sémiotiques et savoir passer des unes aux

XLinguae, Volume 14 Issue 4, October 2021, ISSN 1337-8384, eISSN 2453-711X 
autres ». Le passage évoqué dans la citation susmentionnée n'est possible que grâce à l'activité langagière qui relève de la médiation, celle-ci étant un outil précieux de l'EMILE/CLIL.

Selon le Cadre européen commun de référence pour les langues: apprendre, enseigner, évaluer. Volume complémentaire avec de nouveaux descripteurs (désormais CECR) (Conseil de l'Europe, 2018 : 106), la médiation constitue l'activité langagière dans laquelle "l'utilisateur/apprenant agit comme un acteur social créant des passerelles et des outils pour construire et transmettre du sens soit dans la même langue, soit d'une langue à une autre (médiation interlangues) ». Elle correspond donc le mieux à la spécificité de l'approche EMILE/CLIL, car, dans la médiation, «l'accent est mis sur le rôle de la langue dans des processus tels que créer l'espace et les conditions pour communiquer et/ou apprendre, collaborer pour construire un nouveau sens, encourager les autres à construire et à comprendre un nouveau sens et faire passer les informations nouvelles de façon adéquate " (Conseil de l'Europe, $2018: 106)$.

Le CECR distingue trois types d'activités de médiation, à savoir la médiation de concepts, la médiation de textes et la médiation de la communication, et les définit de manière suivante :

«Médiation de textes signifie transmettre à une personne le contenu d'un texte auquel cette personne n'aurait pas accès, souvent à cause de barrières d'ordre linguistique, culturel, sémantique ou technique.

Médiation de concepts renvoie au processus qui facilite l'accès au savoir et aux concepts, en particulier pour des personnes qui sont incapables d'y accéder directement.

Médiation de la communication. Son objectif est de faciliter la compréhension et de façonner une communication réussie entre les utilisateurs/apprenants qui ont des perspectives individuelles, socioculturelles, sociolinguistiques ou intellectuelles éventuellement différentes » (Conseil de l'Europe, 2018 : 109-110).

Dans le contexte de réflexion qui nous intéresse, il est requis que l'enseignant d'une DNL en LE maîtrise la discipline, la langue de celle-ci, mais aussi la langue spécifique à la communication de la classe. Afin de mettre en exergue la double nature des objectifs d'enseignement (à savoir la langue et la discipline), il est possible d'envisager la définition de la médiation à deux niveaux, comme le font L. Gajo et A.C. Berthoud (2008) en parlant de "médiation» et de « re-médiation ». La médiation consiste à traiter des savoirs disciplinaires et vise prioritairement à conceptualiser le contenu alors que la re-médiation traite des savoirs linguistiques et cherche à clarifier tout ce qui est opaque. Dans une telle perspective, l'enseignant est amené à s'approprier correctement à la fois les propriétés du discours scientifico-pédagogique et les expressions spécifiques pour l'animation de la classe. Qui plus est, sa capacité à (re-)médier ne se limite pas à une compétence linguistique telle quelle, mais elle suppose une mobilisation efficace des stratégies de médiation, soit des " techniques utilisées pour clarifier le sens de ce qui est dit ou écrit et faciliter la compréhension » (Conseil de l'Europe, 2018 : 132). Ces stratégies de médiation étant à la source des gestes professionnels des enseignants devraient être conformes aux conventions, conditions et contraintes du contexte communicatif (ibidem).

Parmi les stratégies de médiation dont l'enseignant peut faire usage dans sa pratique pédagogique, le CECR distingue :

les stratégies pour expliquer un nouveau concept qui consistent à relier à un savoir préalable, décomposer une information, adapter son langage ; 
les stratégies pour simplifier un texte avec deux sous-catégories : amplifier un texte dense et élaguer un texte.

Dans l'approche EMILE/CLIL, les compétences discursives de l'enseignant reposent entre autres sur sa capacité de verbaliser les contenus abordés dans les textes, diagrammes, schémas, mais aussi les définitions, théories, conceptions scientifiques. Ceci amène M. Dudek (2002) à soutenir que l'un des objectifs majeurs de l'enseignement bilingue est de maîtriser le discours scientifique propre à une discipline. Ceci implique naturellement la capacité de définir et de nommer les concepts. Certains sont aussi prêts à admettre (Iluk, 2000) que la fréquence des activités langagières consistant à définir, nommer ou décrire des notions et phénomènes y est très élevée. Nous adoptons ce point de vue pour concentrer notre réflexion sur les gestes professionnels des enseignants des DNL qui concrétisent les stratégies de médiation utilisées au profit de l'explication des nouveaux concepts abordés lors de leçons de chimie et de biologie.

\section{Les gestes professionnels de médiation pour construire le sens}

L'enseignant d'une DNL se sert des stratégies de médiation afin d'aider ses apprenants à comprendre les savoirs et les savoir-dire propres à une discipline. Ces stratégies sont donc étroitement liées aux procédés du traitement d'un contenu original et spécialisé à l'intention des destinataires novices. Nous insistons sur le fait que, dans l'enseignement bilingue, tout savoir disciplinaire et savoir-dire langagier est plus ou moins spécialisé pour l'apprenant, parce qu'il est transmis par l'intermédiaire de la langue qui n'est pas la langue maternelle de l'élève. Il revient donc à l'enseignant-médiateur de se mettre à la recherche des modes d'action les plus optimaux et efficients afin de rendre ce contenu intelligible pour l'apprenant. Doit-il le développer, le simplifier, le reformuler, l'exprimer dans une autre langue ou un autre mode sémiotique, l'illustrer avec des supports visuels, etc. ? Les solutions adoptées lors des pratiques de classe sont variées, ce dont témoignent les exemples enregistrés chez les enseignants de chimie et biologie observés pendant notre recherche.

\subsection{Relier avec un savoir préalable}

Établir des liens avec un savoir préalable constitue un élément inhérent du processus de médiation. Afin de présenter de nouvelles informations, l'enseignant ne peut faire autrement qu'activer les connaissances antérieures des apprenants. L'un des gestes professionnels les plus visibles dans la démarche pédagogique adoptée par les enseignants consiste à « poser des questions pour encourager les personnes à réactiver leurs savoirs préalables " (Conseil de l'Europe, 2018: 132). De tels gestes se manifestent lorsqu'un enseignant fait appel aux contenus déjà abordés en classe et demande aux apprenants de reproduire des connaissances qu'ils ont déjà acquises.

Extrait 1. Leçon de chimie (MK_2C_4-12-20197)

ENS1: Et vous vous souvenez comment on divise les substances, on en a déjà parlé. Ce sont, vous vous souvenez, les corps purs et les mélanges. ...

ENS1: Alors regardez, les oxydes, vous vous souvenez, comment on peut caractériser les oxydes? Qu'est-ce que c'est? ... Zosia est-ce que tu peux dire la définition?

AP : c'est la combinaison de l'oxygène avec un autre élément.

\footnotetext{
${ }^{7}$ Les informations présentées entre parenthèses contiennent les initiales de l'enseignant, la classe (et éventuellement la leçon) ainsi que la date de la leçon et de l'enregistrement.
}

XLinguae, Volume 14 Issue 4, October 2021, ISSN 1337-8384, eISSN 2453-711X 
ENS1: Voilà, donc il y a toujours deux éléments qui constituent un oxyde, oui. Un atome d'oxygène plus un autre élément. Regardez l'image. Vous connaissez déjà pas mal d'oxydes, mais sous quelle forme existent les oxydes?

Extrait 2. Leçon de biologie (MSZ_1L_4-12-2019)

ENS3: Nous allons commencer par un petit rappel. Première loi de Mendel, nous avons appelé la première loi de Mendel, pierwsze prawo Mendla [la première loi de Mendel $]^{8}$, mais en réalité ce n'est pas seulement la première, mais aussi la deuxième. Donc, la première et la deuxième loi de Mendel en français pour donner la première loi de Mendel en polonais. Est-ce que vous vous rappelez de l'expérience de Mendel sur les fleurs? Quelle était cette expérience?

(...)

ENS3: parce que c'est un organisme homozygote, parce que ce sont les lignes pures. Nous les avons croisés avec les lignes pures aussi homozygotes mais quelle est la différence ? Celles-ci sont...

APP: dominants.

ENS3: dominants, et celles-ci sont récessives. Exactement. Donc, qu'est-ce qu'il obtient en F un (F1) ? Qu'est-ce que nous avons dit ? Qu'est-ce qu'il a obtenu?

APP: rouges ?

ENS3: des individus à couleur rouge cent pourcent, n'est-ce pas ? Et quel était leur génotype?

\section{Extrait 3. Leçon de biologie (MZ_2C_4-12-2019)}

ENS2: La caractéristique des ptéridophytes (elle écrit le sujet sur le tableau). La caractéristique des ptéridophytes. Vous connaissez déjà le nom de ce groupe systématique les ptéridophytes. On a cité le nom de ce groupe pendant notre premier cours concernant les végétaux. Donc, je vous demande de rappeler quel est le nom polonais de ce groupe.

(...)

Alors les traits communs de ces plantes c'est déjà la présence des tissus, donc des tissus végétaux caractéristiques (elle écrit sur le tableau) donc présence des tissus végétaux, surtout des tissus vasculaires. Des tissus vasculaires, qui va rappeler quel est le nom polonais ? On a déjà introduit ce terme en parlant de milieu aquatique et de milieu terrestre

Comme en témoignent les extraits 1-3, la façon de construire le discours pédagogique insiste sur la présentation antérieure de certains contenus en classe et suggère, plus ou moins explicitement, l'intégration des savoirs par les apprenants. Les énoncés de l'enseignant sous-entendent l'appropriation des savoirs par les apprenants («vous vous souvenez ») ou affirment l'intégration préalable des contenus (« vous connaissez déjà »), tout en mettant ces derniers avec les sujets déjà traités en classe. Pour parler des composés inorganiques, l'enseignant de chimie réfère à la façon de diviser les substances ou à la forme des oxydes (extrait 1), alors que l'enseignante de biologie cherche à réactiver les connaissances concernant les végétaux pour aborder la caractéristique des ptéridophytes (extrait 3). Parfois, le discours de l'enseignant fait explicitement comprendre que l'étape initiale de la leçon est consacrée au rappel des savoirs préalables (extrait 2). Les énoncés des enseignants que présentent les extraits 1-3 sont marqués par des traces linguistiques spécifiques relatives souvent à des

\footnotetext{
${ }^{8}$ Le texte entre crochets constitue la traduction des expressions polonaises présentes dans les énoncés des enseignants ou ceux des apprenants. Nous utilisons la même forme de transcription de cet élément dans tous les extraits rapportés dans la suite.
} 
marqueurs d'interrogation: «comment», «sous quelle forme », « est-ce que...», «qu'est-ce que...», «quel/quelle» ou d'ordre «je vous demande de...». Dans la plupart des cas, ces marqueurs linguistiques ont pour fonction illocutoire de demander aux apprenants de produire le savoir disciplinaire (extrait 1) ou du vocabulaire (extrait 3 ) ou bien d'expliquer des théories plus complexes (extrait 2).

Cependant on observe aussi des énoncés privés de tels marqueurs linguistiques. L'enseignant incite alors l'apprenant à réactiver son savoir par des ruptures dans le discours pédagogique, accompagnées parfois d'une demande implicite ou explicite d'achèvement (extrait 4). De telles ruptures sont donc intentionnelles, puisque l'enseignant les introduit volontairement afin de faire produire la suite aux apprenants, tout en les amenant à travailler sur les savoirs disciplinaires.

Extrait 4. Leçon de chimie (MK_2C_4-12-2019)

ENS1 : Voilà, donc sous la forme gazeuse, comme par exemple ici l'oxyde d'azote. Sous la forme...

AP : liquide

ENS1 : liquide. Ici c'est quoi ? (il écrit sur le tableau $\mathrm{H}_{2} \mathrm{O}$ )

AP : l'eau

ENS1 : l'eau. Et sous la forme...

AP : $\quad$ stała [solide]

ENS1 : oui, donc sous la forme solide. Vous avez trois formes : la forme gazeuse, la forme liquide et la forme solide. Donc vous voyez que les oxydes peuvent exister sous ces trois formes.

Nous pouvons conclure que les gestes professionnels de médiation utilisés pour établir des liens avec un savoir préalable et présentés dans les extraits 1-4 jouent la fonction de sollicitation. Ils permettent à l'enseignant de stimuler l'apprentissage des apprenants et de les amener à activer leur répertoire de connaissances disciplinaires en LE. De plus, ils remplissent aussi la fonction d'évaluation, puisque la réponse fournie par l'apprenant à la sollicitation de l'enseignant sera évaluée par ce dernier. Cette réponse constitue ainsi un feed-back sur l'efficacité de l'enseignement/apprentissage et donne l'occasion à une mise au point des savoirs relatifs à la discipline et à la langue.

\subsection{Adapter son langage}

L'enseignement d'une discipline non linguistique en langue étrangère permet de développer la conscience linguistique et favorise un travail explicite sur les ressources linguistiques, ces dernières étant en lien direct avec les concepts disciplinaires. La compréhension exacte et l'appropriation correcte des savoirs de la discipline passent, dans l'approche EMILE/CLIL, par le savoir-dire propre et conforme aux domaines de spécialité en LE. Cette caractéristique traduit le besoin de l'enseignant « de faire des changements dans le style et le registre pour inclure le contenu d'un texte à un texte nouveau d'un genre et d'un registre différents. Cela peut se faire à l'aide de synonymes, de comparaisons, de simplifications ou de paraphrases » (Conseil de l'Europe, 2018 : 132).

Extrait 5. Leçon de chimie (MK_2C_L1_4-12-2019)

ENS1: Voilà, donc vous avez en fait quatre caractères. Le caractère numéro un c'était le caractère acide. Ça veut dire quoi ? Un oxyde qui est acide... ça veut dire quoi Bartek?

AP : $\quad$ il est est avec les bases.

XLinguae, Volume 14 Issue 4, October 2021, ISSN 1337-8384, eISSN 2453-711X 
ENS1 : Oui, il réagit avec les bases et il ne réagit pas avec les acides. Voilà, très bien.

(...)

ENS1 : neutre, voilà. Vous avez encore des oxydes neutres. Dominika, ça veut dire quoi ? Un oxyde qui est neutre

AP : $\quad$ il ne réagit pas avec les acides et...

ENS1 : oui, et les bases non plus. Voilà, donc ils sont neutres, ils ne réagissent pas du tout en fait. C'est ça.

Extrait 6. Leçon de chimie (MK_2C_L2_4-12-2019)

ENS1: La combustion c'est la réaction avec quoi ?

AP : Oxygène

ENS1 : L'oxygène, n'est-ce pas. Czyli spalanie [donc la combustion]. Quelque chose qui est combustible cela veut dire que cette chose s'enflamme facilement, oui, brûle facilement. On obtient vous voyez le peroxyde et vous avez la formule de ce composé. Alors le peroxyde ça veut dire quoi en polonais ?

(...)

ENS1 : « solution limpide », co to może być ? [qu'est-ce que cela peut être ?]

AP : roztwór przezroczysty ? [solution transparente]

ENS1 : roztwór przezroczysty, my po chemicznemu często mówimy, że roztwór jest kla-row-ny. Klarowny roztwór, une solution transparente, on peut dire aussi, une solution transparente. [solution transparente. Entre chimistes, nous disons souvent que la solution est claire.]

(...)

ENS : l'oxyde, papier $\mathrm{pH} .$. alcaline, ça veut dire basique. Po polsku też mówimy...// [en polonais on dit aussi souvent//.] En polonais on dit aussi alkaliczny, alkaizować coś.

Extrait 7. Leçon de biologie (MZ_2C_4-12-2019)

ENS2: Et la tige est une tige souterraine. Donc elle se trouve sous le sol.

(...)

ENS2: Les amyloplastes ce sont des plastes qui sont incolores. Très bien. Donc ça c'est un argument, on les trouve dans la pomme de terre qui emmagasine qui stocke / stocker vous vous rappelez ? stocker ou emmagasiner/ de quoi ? La pomme de terre vous m'avez dit stocke de... ?

Extrait 8. Leçon de biologie (MSZ_1L_4-12-2019)

ENS3 : Il a croisé il a fait des croisements il faisait des croisements parce qu'il les faisait pendant des nombreuses années de ces individus, n'est-ce pas. Il les a croisés entre eux. Et qu'est-ce qu'il a obtenu comme résultat Magda en F2?

A partir des exemples rapportés dans les extraits 5-8, il est possible de repérer différentes stratégies de médiation que les enseignants utilisent pour expliquer la signification des mots difficiles et/ou techniques. Tout d'abord, ils sollicitent l'explication du terme par l'apprenant à l'aide d'une question directe et, en fonction de la situation, ils attendent la réponse en langue étrangère (extrait 5) ou maternelle (extrait 3,6). La réponse fournie par l'apprenant est reprise par l'enseignant dans son métadiscours. Ce dernier porte sur la production correcte de l'apprenant : l'enseignant ratifie le choix du vocabulaire et/ou la définition paraphrastique, en la complétant et/ou la reformulant par la suite (extrait 5). Il importe de remarquer que, dans la situation d'enseignement que nous examinons, l'enseignant est considéré comme expert ou possesseur du savoir et savoir-dire spécialisé/disciplinaire. Sa fonction 
consiste donc naturellement à le faire acquérir par les apprenants qui sont novices et se trouvent dans une position d'infériorité par rapport à son niveau de connaissances linguistiques et disciplinaires. Cependant, les questions que l'enseignant adresse aux élèves peuvent être qualifiées de questions «simulées » et "fictives » : l'enseignant fait semblant de ne pas connaître le terme spécialisé afin de le faire produire par les apprenants. Les extraits des interactions en classe rapportés ci-dessus mettent ainsi en avant la spécificité de la situation pédagogique où se superposent deux énonciations (Trévise, 1979: 44) : l'énonciation véritable de la classe et l'énonciation translatée dans laquelle l'enseignant s'identifie à un individu fictif qui ne possède pas le savoir requis. Par ailleurs, les enseignants recourent aux synonymes ou à la paraphrase pour formuler des définitions ou expliquer eux-mêmes les termes en langue étrangère (extraits 6,7 et 8). Ces techniques sont intégrées dans le discours pédagogique qui est alors formulé par l'enseignant entièrement en langue étrangère et privilégie souvent la présentation monologale.

Les exemples susmentionnés (extrait 6) nous amènent aussi à formuler une autre observation liée au statut de la L1 dans le métadiscours de l'enseignant. La langue maternelle des apprenants accomplit souvent un rôle pivot dans la construction des savoirs langagiers et disciplinaires en L2. En reformulant en L1 des énoncés ou termes de L2 et inversement, les enseignants cherchent à maintenir l'activité métalinguistique des apprenants en deux langues. Ceci est particulièrement repérable dans les explications et commentaires pédagogiques ayant trait à la terminologie et au fonctionnement de la langue, ce qu'illustre entre autres l'extrait 9.

Extrait 9. Leçon de chimie (MK_2C_L1_4-12-2019)

ENS1: Les sel des acides non oxygénés c'est le suffixe -ure. Tak, czyli po polsku dawaliśmy w tych przypadkach w tych solach jaką koncówkę ? [Oui, quel était le suffixe que nous avons utilisé pour ces sels en polonais?]

AP : $\quad-$ ek.

ENS1 : -ek. Chlorek, bromek, siarczek, jodek, fluorek itd. A tu będzie -ure. Natomiast jeśli po polsku dajemy koncówke -an, to tu będzie -ate. I tak na przykład proszę zobaczyć (il lit le slaid) chlorure de potassium, bromure d'argent i tak dalej i tak dalej. Ale już sulfate six de cuivre, sulfate quatre de potassium, carbonate de sodium. [-ek. Chlorure, bromure, sulfure, iodure, fluorure etc. Si en polonais vous utilisez le suffixe -an, en français ça donne-ate. Regardez. (il lit le slaid) chlorure de potassium, bromure d'argent et ainsi de suite. Mais attention sulfate six de cuivre, sulfate quatre de potassium, carbonate de sodium ...]

Même si certaines paraphrases en mode bilingue donnent parfois lieu à une simplification des contenus, il importe aussi de constater que les enseignants attachent une attention particulière à ce que les apprenants se servent d'un vocabulaire adéquat à la discipline lorsqu'ils s'expriment en L1 ou L2 (extrait 11) et au contexte de son apparition/usage (la communauté professionnelle des chimistes - extrait 6 ; la classe extrait 10).

Extrait 10. Leçon de biologie (MSZ_1L_4-12-2019)

ENS3: Et on s'était arrêté là pour la première loi de Mendel. Tous les individus cent pourcent nous les savons tous les individus en F1 sont hétérozygotes et ont le même caractère, dirait Mendel parce que Mendel ne parlait pas de gène, ne parlait pas d'allèle, il parlait de caractère. Donc tous les individus en $F 1$, tous ces individus-là sont hétérozygotes et ont le même caractère, c'est-à-dire sont rouges. Ça serait la première loi de Mendel en français.

XLinguae, Volume 14 Issue 4, October 2021, ISSN 1337-8384, eISSN 2453-711X 
Et qu'est-ce qu'il a fait Mendel pour obtenir F deux (F2) ? Quelle était l'étape entre F1 et F2, qu'est-ce qu'il a fait en utilisant le verbe croiser ? QU'est-ce qu'il a fait?

APP : il croisait les fleurs de l'étape F1 et (incompréhensible)

ENS3 : Oui, ce que tu as dit c'est tout à fait ce que j'attendais. Il croisait les fleurs de la génération F1, donc il croisait ces fleurs-là entre elles, pas les fleurs, les individus, mais enfin les fleurs c'est notre façon de parler.

\section{Extrait 11. Leçon de biologie (MZ_2C_4-12-2019)}

ENS2: kupki zarodni, w sensie skupiska. [un amas de sporanges, au sens de groupement] Donc, vous pouvez tout de suite oublier ce mot c'est un amas.

Vous retenez des sporanges. Le mot sporange ça va, mais un amas de sporanges c'est une exagération, c'est juste pour pouvoir en parler.

En analysant les gestes professionnels de médiation, il est possible de noter que les enseignants de discipline non seulement explicitent des notions disciplinaires, mais aussi mettent régulièrement en relief l'existence de différents registres discursifs, ce qui les amène aux stratégies de contextualisation et décontextualisation de ces notions. La langue telle qu'elle fonctionne dans la discipline joue un rôle de distanciation vis-à-vis de l'expérience dans d'autres contextes de la vie (extraits 12 et $13)$.

Extrait 12. Leçon de biologie (MSZ_1L_4-12-2019)

ENS 3: Donc, à partir de ça, Mendel dit et formule ce qu'on appelle la deuxième loi de Mendel pour les Français, (elle écrit sur le tableau) deuxième loi de Mendel qui est la loi de pureté des gamètes et c'est ce qu'on appelle en polonais première loi de Mendel pierwsze prawo Mendla prawo czystości gamet. A co to jest gameta? Myśmy na razie tego słowa nie użyli.../ czystości [Et qu'est-ce que c'est un gamète? Nous n'avons pas utilisé ce mot jusqu'à présent.../ pureté] pureté/ pur on peut utiliser ce mot dans différents sens. Ça peut être l'air, aujourd'hui nous avons beaucoup de soleil n'est-ce pas cela veut dire que l'air est pur, l'atmosphère est pure czyli... [c'est-à-dire...]

APP: $\quad$ powietrze czyste [l'air pur]

ENS3: jak powiemy l'air est pur, l'atmosphère est pure to będziemy to odnosić do powietrza, tzn. że nie jest ono zanieczyszczone. To słowo [Quand on dit l'air est pur on fait référance à l'air pour dire qu'il n'est pas pollué. Ce $m o t$ ] pureté ça peut être aussi une personne, une fille. Au Moyen Age, on dirait elle était pure czysta w zupełnie innym znaczeniu dziewictwa. Tak że to jest słowo, które ma różne znaczenia, ale tutaj gamety są czyste. Co to znaczy, że gamety są czyste? [dans un autre sens, virginité. C'est un mot qui a des significations différentes, et ici ce sont les gamètes qui sont pures. Qu'est-ce que cela veut dire que les gamètes sont pures?]

APP : może, że nie są wymieszane [Peut-être qu'ils ne sont pas mélangés]

ENS3 : bardzo dobrze, czyli zawierają zawsze tylko jeden... jakie słowo ?... na a...[Très bien, c'est-à-dire qu'ils ont un seul... quel mot ?... il commence avec un , $a, \ldots]$

APP : allel [allèle]

ENS3 : allel, oczywiście. Mendel by powiedział charakter, cecha, ale my mówimy, że tylko jeden allel, bo to co sobie piszemy to dlatego, że to może się połączyć z tym [un allèle, bien sûr. Mendel aurait dit un caractère, un trait, mais nous disons qu'ils ont un allèle, parce que ce que nous écrivons c'est pour pouvoir le mettre en relations avec ceci] 
De plus, la manière de réaliser différents actes de paroles peut varier selon les langues, ce qui risque de faire apparaître des interférences d'usage des termes par les enseignants et/ou apprenants. Certains termes ou notions fonctionnent différemment dans le discours disciplinaire en $\mathrm{L} 1$ et $\mathrm{L} 2$ ou bien ils réfèrent à des réalités distinctes (extraits 13,14). Dans de tels cas, le commentaire pédagogique de l'enseignant est indispensable afin d'attirer l'attention de l'apprenant sur la façon dont les concepts fonctionnent dans le discours de la discipline, d'expliquer la source des divergences et, éventuellement, de préciser les conséquences d'un usage imprécis/incorrect.

\section{Extrait 13. Leçon de biologie (MSZ_1L_4-12-2019)}

ENS 3: Aujourd'hui nous allons travailler sur la deuxième loi de Mendel, qu'on appelle en français la troisième loi de Mendel. Donc pour nous Polonais on va dire które prawo Mendla [quelle loi de Mendel?]? Drugie [deuxième], voilà, drugie prawo Mendla [deuxième loi de Mendel], mais les Français l'appellent la troisième loi de Medel. Donc, contradiction.

Nous allons commencer par un petit rappel. Première loi de Mendel, nous avons appelé la première loi de Mendel, pierwsze prawo Mendla [premi ère loi de Mendel], mais en réalité ce n'est pas seulement la première, mais aussi la deuxième. Donc, la première et la deuxième loi de Mendel en français pour donner la première loi de Mendel en polonais.

Extrait 14. Leçon de biologie (MŻ_1S_4-12-2019)

ENS2: Donc, qu'est-ce qu'il faut mettre? Comment on l'appelle? Rappelez. Jodyna?

APP : iodine ?

ENS2: ce serait le plus simple, mais c'est pas ça. C'est l'eau iodée. W zasadzie mamy tutaj pewien problem językowy. Bo de facto skoro jest to woda $\mathrm{z}$ jodem, to tak naprawdę po prostu nazywano to płynem Lugola. My natomiast nie używamy roztworu wodnego tylko alkoholowego nazywanego jodyną. To dlatego, że jest to trwalsze. Płyn Lugola jest nietrwały i musielibyśmy co chwila tego jodu tam rozpuszczać, żeby bez przerwy mieć odczynnik. Natomiast jodyna może sobie stać, stąd używamy jodyny. Ale de facto jest to ten sam odczynnik, bo chodzi nam o jod już niezależnie od tego w jakim roztworze. [En fait, nous avons ici un problème linguistique, parce qu'il s'agit de facto de l'eau avec de l'iode, qu'on appelait simplement la solution de Lugol. En revanche nous, on n'utilise pas de solution aqueuse, mais une solution alcoolique appelée l'eau iodée. C'est parce que ses propriétés sont plus durables. Le liquide de Lugol est instable et nous devrions dissoudre de l'iode de temps en temps pour avoir le même réactif en continu. Cependant, l'eau iodée peut conserver longtemps ses propriétés et c'est pourquoi nous l'utilisons. Mais en fait, c'est le même réactif, car il $s$ 'agit de l'iode quelle que soit la solution.]

La reformulation constitue l'une des stratégies courantes auxquelles recourent les enseignants adoptant l'approche EMILE/CLIL. Il reste pourtant intéressant d'insister sur le fait que les procédés de la reformulation sont variés et évoluent au cours de la leçon en fonction des objectifs pédagogiques visés. Comme nous l'avons déjà démontré, ils comprennent les alternances codiques (extraits 3,6), la paraphrase ou le «savoir-dire autrement" au sein de la même langue (extraits 1, 5-8) ou bien les relations entre une notion disciplinaire et une situation de la vie quotidienne (extrait 12). Il convient d'y ajouter les procédés de reformulation qui prennent en compte les changements de modes sémiotiques, comme dans les extraits ci-dessous (15-16).

XLinguae, Volume 14 Issue 4, October 2021, ISSN 1337-8384, eISSN 2453-711X 
Extrait 15. Leçon de chimie (MK_2C_L2_4-12-2019)

ENS1: Écrivez sous forme moléculaire l'équation de la réaction qui s'est produite pendant l'expérience décrite - ajout d'oxyde de métal à de l'eau, $3^{\mathrm{e}}$ réaction. C'est quel oxyde de métal Bartek?

AP : $\quad \mathrm{Na}_{2} \mathrm{O}$.

ENS1: Donc? Quel oxyde?

AP : Oxyde de sodium.

ENS1 : Oxyde de sodium, voilà. Qui veut bien écrire la réaction? Bartek, viens au tableau. Donc, Filip c'est à toi l'exercice suivant.

AP : $\quad$ (il écrit au tableau) $\mathrm{Na}_{2} \mathrm{O}+\mathbf{H}_{2} \mathrm{O}->\mathbf{2} \mathbf{N a O H}$

Extrait 16. Leçon de biologie (MŻ_1S_4-12-2019)

ENS2 : Alors, qu'est-ce qu'on observe?

APP : la couleur [se $\left.\int \tilde{a} z \mathrm{e}\right]$ à violet.

ENS2: Alors, la preuve définitive que ce sont des amyloplastes, ils sont violets plutôt violets très bien (elle continue d'écrire sur le tableau) et deviennent et deviennent// donc amyloplastes incolores stockent de l'amidon et deviennent violets à la présence d'eau iodée. Donc ce sont des plastes ou des organismes incolores qui stockent de l'amidon et qui deviennent violets à la présence d'eau iodée. Donc c'est la preuve définitive que ce sont des amyloplastes.

Le passage d'un mode sémiotique à un autre est particulièrement valable pour le contexte d'enseignement des sciences naturelles (telles que la chimie ou la biologie) où l'enseignant et/ou l'apprenant sont souvent amenés à verbaliser une expression symbolique d'éléments (extrait 15), une schématisation (fléchage, dessin, schéma etc.) ou bien s'exprimer à partir d'objets matériels, ne serait-ce que lors d'une expérimentation (extrait 16). Les gestes professionnels de médiation utilisés par les enseignants stimulent l'activité cognitive des apprenants tout en les incitant à formuler l'information disciplinaire en différents modes sémiotiques en vigueur et à expliciter les résultats des découvertes scientifiques réalisées en classe.

\subsection{Décomposer une information compliquée}

Enfin, le travail sur l'appropriation des nouveaux concepts peut amener l'enseignant à recourir au troisième type de stratégies de médiation distingué par le CECR (Conseil de l'Europe, 2018 : 133), soit à décomposer «une information compliquée en ses parties constituantes ». Les gestes professionnels de l'enseignant visent alors à disséquer un ensemble complexe d'informations en une série d'éléments isolés qui forment un tout cohérent. Voici une épreuve du baccalauréat bilingue auquel se préparent les apprenants.

\section{Extrait 17. Leçon de chimie (MK_2C_L2_4-12-2019)}

« La combustion du sodium dans 1 'air a pour produit non pas l'oxyde mais le peroxyde de ce métal, de formule Na2O2 (1 ère réaction). Pour obtenir de l'oxyde de sodium, il faut, à température élevée, effectuer la $2^{\mathrm{e}}$ réaction illustrée par l'équation suivante :

$$
\mathrm{Na} 2 \mathrm{O} 2+2 \mathrm{Na} \rightarrow 2 \mathrm{Na} 2 \mathrm{O}
$$

La $2^{\mathrm{e}}$ réaction a été réalisée en laboratoire, ensuite on a effectué l'expérience illustrée ci-dessous.

oxyde métallique solide obtenu dans la $2^{\mathrm{e}}$ réaction.

$\mathrm{Au}$ cours de l'expérience, une transformation chimique a été effectuée ( $3^{\mathrm{e}}$ réaction). Une solution limpide incolore s'est formée dans le tube à essai et son contenu est plus chaud qu'avant le mélange des réactifs. Ensuite, dans la 
solution obtenue, un papier indicateur universel jaune a été immergé, qui a changé sa couleur en bleu-vert.

Écrivez sous forme moléculaire l'équation de la $1^{\text {ère }}$ réaction.

Écrivez sous forme moléculaire l'équation de la $3^{\mathrm{e}}$ réaction ».

Afin que les apprenants s'acquittent correctement de la tâche demandée, l'enseignant décompose leur travail en une série d'étapes au cours desquelles il cherche à vérifier le niveau de leur compréhension de l'épreuve tout en orientant leur attention sur la réponse appropriée. Il aborde séparément les points principaux de la consigne relatifs aussi bien à la discipline qu'à la langue. Au fur et à mesure des sollicitations directes effectuées en mode bilingue (extrait 6), l'enseignant incite les apprenants à réactiver leurs connaissances disciplinaires (la réaction de combustion, les propriétés des peroxydes) et linguistiques (solution limpide) et à les investir dans la formulation autonome de la réponse définitive (extrait 15). Les stratégies de médiation visant à simplifier la complexité de l'effort cognitif des apprenants face à un texte aident en même temps les apprenants à respecter le raisonnement disciplinaire et sa méthodologie. Une telle analyse progressive et détaillée du problème à résoudre rend les apprenants capables de percevoir les liens entre différents éléments de l'expérimentation, de mieux comprendre leurs effets réciproques et de réussir finalement dans la résolution du problème.

\section{Conclusion}

Les gestes professionnels de médiation, que nous avons décrits à travers les extraits des pratiques de classe choisies, sont - à notre avis - suffisants pour constater que le savoir disciplinaire «s'acquiert par la participation à des expériences médiées dans la communication avec un expert »(Carol, 2015 : non paginé). Dans l'enseignement bilingue, il s'agit des expériences et de la communication vécues dans la salle de classe où le statut de l'expert est assigné à l'enseignant qui intervient dans différents champs d'action, à savoir la discipline, la langue, la didactique. L'approche EMILE/CLIL consiste à enseigner une matière scolaire en langue étrangère, mais celle-ci constitue à la fois un outil d'enseignement et d'élaboration de concepts. La communication en classe se réalise certes à travers les signes linguistiques, les actes de paroles et les activités langagières, mais le rôle de ces derniers est d'assurer l'abstraction, la généralisation, la simplification, la catégorisation ainsi que la systématisation des significations. Toutes ces opérations effectuées à l'intermédiaire de la langue se matérialisent dans le discours pédagogique de l'enseignant (soit son métadiscours) sous forme des gestes professionnels de médiation utilisés à différentes étapes de l'enseignement.

Suite à notre analyse, nous parvenons à certains constats relatifs à l'abondance de stratégies de médiation mobilisées dans une situation d'enseignement assurée selon les principes de l'approche EMILE/CLIL. Nous insistons sur la présence du savoir langagier et métalangagier qui intervient en permanence dans la transmission des contenus disciplinaires sous forme de métadiscours ou bien d'énonciation transposée. La transmission des connaissances à enseigner/apprendre se fait par des va-et-vient i) entre la langue maternelle (L1) et la langue étrangère (LE/L2), ii) entre différents modes sémiotiques, iii) entre différentes cultures disciplinaires, iv) entre différents contextes (spécialisés et non-spécialisés) donnant l'opportunité de vulgariser le savoir disciplinaire et/ou spécialiser le savoir ordinaire. Le métalangage y joue un rôle important et précieux. Nous en concluons que la prise en compte de la discipline et la langue qui constituent l'essence de l'approche EMILE/CLIL n'est point possible si l'enseignant ne sait pas se servir de stratégies de médiation grâce auxquelles il est à

XLinguae, Volume 14 Issue 4, October 2021, ISSN 1337-8384, eISSN 2453-711X 
même de traiter le savoir disciplinaire de manière opérationnelle, de rendre ce dernier intelligible au destinataire et d'aider celui-ci à se l'approprier correctement.

\section{Bibliographic references}

CAROL, R. 2015. La didactique de l'enseignement bilingue : enseignement bilingue et langage. In : Recherches en didactique des langues et des cultures, n. 12-3, Available online: http://journals.openedition.org/rdlc/973 ; DOI : https://doi.org/10.4000/rdlc.973. ISSN : 1958-5772.

CENOZ, J. - GORTER, D. 2010. The Diversity of Multilingualism in Education. In: International Journal of the Sociology of Language, n. 205, pp. 37-53. DOI: 10.1515/IJSL.2010.038. ISSN: 0165-2516

CHISS, J.-L. 2012. Postface. In : Causa, M. (ed.) : Formation initiale et profil d'enseignant de langue, Enjeux et questionnement. Bruxelles: De Boeck. pp. 317320. ISBN : 9782804171292

CONSEIL DE L'EUROPE. 2001. Cadre Européen Commun de référence pour les langues : Apprendre, Enseigner, Évaluer. Paris : Didier. ISBN : 9782278058136

CONSEIL DE L'EUROPE. 2018. Cadre Européen Commun de référence pour les langues : Apprendre, Enseigner, Évaluer. Volume complémentaire avec de nouveaux descripteurs, Available online : https://rm.coe.int/cecr-volume-complementaire-avecde-nouveaux-descripteurs/16807875d5. ISBN non disponible

COYLE, D. - HOOD, P. - MARSH, D. 2010. CLIL: Content and Language Integrated Learning. Cambridge: Cambridge University Press. ISBN: 9780521130219 COYLE, D. 2006. Content and Language Integrated Learning - Motivating Learners and Teachers. In: The Scottish Language Review, n. 13, pp. 1-18, Available online: http://www.strath.ac.uk/scilt/slr/iss/2006/13/. ISSN non disponible

COYLE, D. 2007. Content and Language Integrated Learning: Towards a connected research agenda for CLIL pedagogies. In: The International Journal of Bilingual Education and Bilingualism, vol. 10, n. 5, pp. 543-562. ISSN: 13670050

DALTON-PUFFER, C. - LLANARES, A. - LORENZO, F. - NIKULA, T. 2014. You Can Stand Under My Umbrella: Immersion, CLIL and Bilingual Education. A Response to Cenoz, Genesee \& Gorter. In: Applied Linguistics, vol. 35, n. 2, pp. 213218. ISSN: 0142-6001

DALTON-PUFFER, C. 2011. Content and Language Integrated Learning: From Practice to Principles? In: Annual Review of Applied Linguistics, n. 31, pp. 182-204. ISSN: 0267-1905

DUDEK, M. 2002. Kwalifikacje nauczycieli w nauczaniu bilingwalnym. In: Języki Obce w Szkole, n. 6, pp. 37-40. ISSN: 0446-7965

ESCOBAR URMENETA, C. 2011. Colaboración interdisciplinar, Partenariado y Centros de Formación Docente: Tres ejes para sustentar la formación del profesorado AICLE. In: Escobar Urmeneta C. - Nussbaum, L. (eds.): Aprendre en una altra llengua / Learning through another language / Aprender en otra lengua. Bellaterra: Servei de Publicacions de la Universitat Autònoma de Barcelona. pp. 203-230. ISBN: 978-84-490-2677-5

EURYDICE. 2006. L'enseignement d'une matière intégré à une langue étrangère (EMILE) à l'école en Europe. Bruxelles. ISBN : 92-79-00581-2

GAJO, L. - BERTHOUD, A.-C. 2008. Rapport final, Construction intégrée des savoirs linguistiques et disciplinaires dans l'enseignement bilingue au secondaire et au tertiaire, Diversité des langues et compétences linguistiques en Suisse, programme national de recherche PNR 56. ISBN non disponible.

GARCIA, O. - BAETENS BEARDSMORE, H. 2009. Bilingual Education in the 21st Century: a Global Perspective. Oxford: Wiley-Blackwell Pub. ISBN: 978-1-40511994-8

GOZDAWA-GOLEBIOWSKI, R. - NAWROT-LIS, B. - OPACKI, M. SKOCZYLAS, K. 2019. State of the Content and Language Integrated Learning 
(CLIL): implementation in vocational schools across Europe. In: Polish Journal of Continuing Education, n. 3(106), pp. 169-189. eISSN 1507-6563

ILUK, J. 2000. Nauczanie bilingwalne. Modele, koncepcje, założenia metodyczne. Katowice: Wydawnictwo Uniwersytetu Śląskiego. ISBN: 83-226-1004-1

MARSH, D. - MALTJERS, A. - HARTIALA, A.-K. (eds) 2001. Profiling European CLIL Classrooms - Languages Open Doors. Jyväskylä: University of Jyväskylä. ISBN: 9513908623

MARSH, D. - MARSLAND, B. (eds) 1999. Learning with Languages. A professional Development Pro-gramme for Introducing Content and Language Integrated Learning. Finland: University of Jyväskylä. ISBN: 951-39-0578-0

MET, M. 1999. Content-based instruction: Defining terms, making decisions. NFLC Reports, Washington, DC: The National Foreign Language Center, Available online https://carla.umn.edu/cobaltt/modules/principles/decisions.pdf. ISBN/ISSN non disponible.

OLPINSKA-SZKIELKO, M. 2008. Nauczanie dwujęzyczne w świetle badań i koncepcji glottodydaktycznych. Warszawa: Euroedukacja. ISBN: 978-83-64020-09-4 PEGOURIE-KHELLEF, M. 2019. Les gestes professionnels des enseignants de disciplines dites non linguistiques dans trois établissements à dispositif d'enseignement bilingue français-arabe en Egypte. Université de Rennes 2. NNT : 2019REN20028. HAL-id : tel-02389080. ISBN/ISSN non disponible

RODRIGUES, C. - WIGHAM, C.R. 2013. L'aide linguistique dans l'approche de l'EMILE : pour un équilibre entre compétences langagières et disciplinaires. In : Recherche et pratiques pédagogiques en langues de spécialité, vol. 32, n. 3, Available online : $\quad$ http://journals.openedition.org/apliut/3878; $\quad$ DOI : https://doi.org/10.4000/apliut.3878. ISSN: 2119-5242.

TREVISE, A. 1979. Spécificité de l'énonciation didactique dans l'apprentissage de l'anglais par des étudiants francophones. In : Encrages, no spécial de linguistique appliquée, pp. 44-52. ISSN non disponible

WHITTY, G. 1996. Creating Quasi-Markets in Education: A review of recent research on Parental choice and school autonomy in three countries. In: Review of Research in Education, n. 22, pp. 89-95. DOI: https://doi.org/10.3102/0091732X022001003. ISSN: 0091-732X

WOLFF, D. 2002. Tworzenie curriculum i kształcenie nauczycieli w dwujęzycznym nauczaniu przedmiotów niejęzykowych. In: Dakowska, M. - Olpinska, M. (eds.): Edukacja dwujęzyczna. Przedszkole, szkoła podstawowa i średnia. Teraźniejszość i Przyszłość. Warszawa: Wydawnictwo Uniwersytetu Warszawskiego. pp.128-153. ISBN: 978-83-64020-21-6

Words: 9359

Characters: 62352 (34,64 standard pages)

Dr hab. Magdalena Sowa, prof. UMCS

University Maria Curie-Skłodowska

Pl. Marii Curie-Skłodowskiej 4a, 20-031 Lublin

Poland

magdalena.sowa@umcs.pl

Dr Joanna Kic-Drgas

University Adam Mickiewicz

Al. Niepodległości 4, 61-874 Poznań,

Poland

j.drgas@amu.edu.pl

XLinguae, Volume 14 Issue 4, October 2021, ISSN 1337-8384, eISSN 2453-711X 